\title{
Anomaly-free leptophilic axionlike particle and its flavor violating tests
}

\author{
C. Han, ${ }^{1, *}$ M. L. López-Ibáñez $\oplus^{2, \dagger}$ A. Melis, ${ }^{3, \star}$ O. Vives ${ }^{4, \S}$ and J. M. Yang ${ }^{2,5, \|}$ \\ ${ }^{1}$ School of Physics, Sun Yat-Sen University, Guangzhou 510275, China \\ ${ }^{2}$ CAS Key Laboratory of Theoretical Physics, Institute of Theoretical Physics Chinese Academy of Sciences, \\ Beijing 100190, China. \\ ${ }^{3}$ Laboratory of High Energy and Computational Physics, National Institute of Chemical Physics \\ and Biophysics, Rävala pst. 10, 10143 Tallinn, Estonia \\ ${ }^{4}$ Departament de Física Tèorica, Universitat de València \& IFIC, Universitat de València \& CSIC, \\ Dr. Moliner 50, E-46100 Burjassot (València), Spain \\ ${ }^{5}$ School of Physical Sciences, University of Chinese Academy of Sciences, Beijing 100049, China
}

(Received 17 August 2020; accepted 9 February 2021; published 25 February 2021)

\begin{abstract}
Motivated by the recent Xenon1T result, we study a leptophilic flavor-dependent anomaly-free axionlike particle (ALP) and its effects on charged-lepton flavor violation. We present two representative models. The first one considers that the ALP origins from the flavon that generates the charged-lepton masses. The second model assumes a larger flavor symmetry such that more general mixings in the charged-lepton are possible, while maintaining flavor-dependent ALP couplings. We find that a keV ALP explaining the Xenon1T result is still viable for lepton flavor violation and stellar cooling astrophysical limits. On the other hand, if the Xenon1T result is confirmed, future charged-lepton flavor violation measurements can be complementary to probe such a possibility.
\end{abstract}

DOI: 10.1103/PhysRevD.103.035028

\section{INTRODUCTION}

Recently, the Xenon collaboration reported the observation of an excess in the electron recoiling energy around the keV scale in the Xenon1T detector [1]. Shortly after its announcement, a lot of theoretical work has been done to interpret the results in the context of different extensions of the Standard Model, like axionlike particles (ALPs) [2-10], dark matter [11-35], neutrinos [36-45] and solar axions [46-48], which, however, are subject to stringent constraints from stellar cooling [49-51].

In this work we focus on the ALP framework to explain this excess. This scenario assumes the existence of an ALP as a sizeable part of the observed dark matter (DM) abundance [2], with a mass of a few $\mathrm{keV}$ and a relatively weak coupling to the electron. However, constraints from $\mathrm{x}$-ray observations forbid the existence of an anomalous coupling of the ALP to photons for $m_{a} \gtrsim 0.1 \mathrm{keV}$.

\footnotetext{
*hanchch@mail.sysu.edu.cn

maloi2@uv.es

¥aurora.melis@uv.es

§oscar.vives@uv.es

jmyang@itp.ac.cn
}

Published by the American Physical Society under the terms of the Creative Commons Attribution 4.0 International license. Further distribution of this work must maintain attribution to the author(s) and the published article's title, journal citation, and DOI. Funded by SCOAP .
Therefore, we are forced to consider an anomaly-free ALP with respect to $\mathrm{U}(1)_{\mathrm{em}}$, to avoid these bounds. With the SM fermionic particle content (plus right-handed neutrinos), only hypercharge and $B-L$ are completely anomaly free with family universal charges. Hypercharge can be immediately discarded as it cannot be broken above the electroweak scale. The breaking of $B-L$ gives rise to a pseudo-Goldstone boson, the Majoron, coupling only to neutrinos at tree level. Although it couples to charged leptons at one-loop level, the $B-L$ breaking scale is related to the right-handed Majorana masses that is necessarily too high to explain Xenon1T result with our minimal particle content. Lepton number, also anomaly free with respect to electromagnetism, faces the same problem with family universal charges.

Thus, we have to consider an anomaly-free U(1) symmetry with family-dependent charges which, as we will see, necessarily implies flavor-changing couplings between the ALP and the SM-lepton sector. If this excess is confirmed in the future, it will be necessary to investigate the lepton flavor violating signatures of this particle in low-energy experiments. In this paper, we consider the flavor violation effects induced by such anomaly-free ALP, and we show that lepton flavor violating (LFV) measurements are essential to probe this possibility.

Our paper is organized as follows: in Sec. II, we present two different models, both flavor dependent, but with distinct mixing patterns; the most important constraints to our models are collected in Sec. III; in Sec. IV, we 
present our results and discuss how these flavored models can be tested by LFV data; finally, Sec. V is dedicated to our conclusions.

\section{MODELS}

We consider a $\mathrm{U}(1)_{\phi}$ global symmetry spontaneously broken by the vev of a complex scalar field, $\phi$, whose angular component is identified with an ALP. We propose two models with flavor dependence on the lepton sector and evaluate the importance of present and future experiments on LFV decays. In the first model, the presence of the ALP is directly connected to the SM flavor puzzle and the breaking of the $\mathrm{U}(1)_{\phi}$ is the only responsible of the observed hierarchy among the lepton generations. Instead, model II generalizes the previous structure assuming the existence of a larger symmetry, which includes $\mathrm{U}(1)_{\phi}$ as a subgroup, whose breaking produces the Yukawa structures at high energies. In this way, we can partly decouple the nonanomalous flavor-dependent $\mathrm{U}(1)_{\phi}$ charges from the observed leptonic masses and mixings. In both models, below the $\mathrm{U}(1)_{\phi}$-breaking scale, the ALP has flavordependent couplings.

\section{A. Model I: hierarchical mixing}

Flavor symmetries à la Froggatt-Nielsen [52] offer an attractive solution to the origin of the observed hierarchy among the charged-fermion families. In its simplest version, the spontaneous breaking of a U(1) flavor symmetry by the vev of a scalar field, usually called flavon, generates it as powers of the ratio between its vev, $v_{\phi}$, and $\Lambda$, the scale at which the heavy fields mediating the processes live, $\epsilon=\left|v_{\phi} / \Lambda\right|$.

In model I, we identify this symmetry with the global $\mathrm{U}(1)_{\phi}$ so that the angular component of the flavon corresponds to the ALP. The case of the anomalous QCD axion has been previously explored in [53,54], with the scalar receiving the name of flaxion or axiflavon.

As usual, in flavor models, distinct mixing patterns can be derived for different charge assignments. Here we focus on the leptonic sector, hence quarks are assumed to be uncharged under the symmetry. Besides, a sufficient condition to obtain an electromagnetic anomaly-free ALP is

$$
\sum_{i} Q_{L_{i}}=0, \quad \sum_{i} Q_{e_{i}}=0,
$$

where $Q_{L_{i}}$ and $Q_{e_{i}}$ are the charges of the left-handed and right-handed charged leptons under the $\mathrm{U}(1)_{\phi}$. Choosing them as $L(1,0,-1)$ and $e(-1,0,1)$, the desired FroggattNielsen structure can be generated.

Two Higgs doublets are introduced with charge 0 and -2 , and an additional $Z_{2}$ symmetry is imposed as in the type- $X$ 2HDM so that the only odd fields are $\mathrm{H}_{2} \rightarrow-\mathrm{H}_{2}, e \rightarrow-e$ and $N_{R} \rightarrow-N_{R}[55,56]$. The Higgses, $H_{1}$ and $H_{2}$, couple to quarks and leptons, respectively. To summarize, the following particles and charges under $\mathrm{U}(1)_{\phi} \times Z_{2}$ are considered for model I:

$$
\begin{aligned}
& H_{1}(0 ; 1), H_{2}(2 ;-1), \phi(1 ; 1), \\
& L(1,0,-1 ; 1), e(-1,0,1 ;-1), N_{R}(0,0,0 ;-1) .
\end{aligned}
$$

From (2), it can be seen that the anomalies cancel for both the left- and right-handed sector. The most general scalar potential is

$$
\begin{aligned}
V\left(H_{1}, H_{2}, \phi\right)= & m_{1}^{2} H_{1}^{\dagger} H_{1}+m_{2}^{2} H_{2}^{\dagger} H_{2}+\lambda_{1}\left(H_{1}^{\dagger} H_{1}\right)^{2} \\
& +\lambda_{2}\left(H_{2}^{\dagger} H_{2}\right)^{2}+\lambda_{3}\left(H_{1}^{\dagger} H_{1}\right)\left(H_{2}^{\dagger} H_{2}\right) \\
& +\lambda_{4}\left|H_{1} \cdot H_{2}\right|^{2}+m^{2} H_{1} \cdot H_{2} \\
& +\lambda\left(\phi^{\dagger} \phi-v_{\phi}^{2}\right)^{2},
\end{aligned}
$$

where the soft term $m^{2} H_{1} H_{2}$, with $m \sim \mathcal{O}(\mathrm{EW})$, breaks explicitly the $\mathrm{U}(1)_{\phi} \times Z_{2}$ symmetry and contributes to the ALP mass after the EWSB. The corresponding Yukawa terms are

$$
\begin{gathered}
\mathcal{L}_{Y} \supset Y_{u} \bar{Q} \tilde{H}_{1} u+Y_{d} \bar{Q} H_{1} d+c_{i j}^{e} e^{n_{i j}^{e}} \bar{L}_{i} \tilde{H}_{2} e_{j} \\
+c_{i j}^{\nu} \epsilon^{n_{i j}^{\nu}} \bar{L}_{i} H_{2} N_{j}+\left(M_{R}\right)_{i j} N_{R_{i}} N_{R_{j}}^{c},
\end{gathered}
$$

with $c_{i j}^{e}$ and $c_{i j}^{\nu} \mathcal{O}(1)$ coefficients and $n_{i j}^{e}=q_{L_{i}}-q_{e_{j}}+$ $q_{H_{2}}, n_{i j}^{\nu}=q_{L_{i}}-q_{N_{R, j}}-q_{H_{2}}$. In model I, we have

$$
n_{i j}^{e}=\left(\begin{array}{lll}
4 & 3 & 2 \\
3 & 2 & 1 \\
2 & 1 & 0
\end{array}\right), \quad n_{i j}^{\nu}=\left(\begin{array}{lll}
1 & 1 & 1 \\
2 & 2 & 2 \\
3 & 3 & 3
\end{array}\right) \text {. }
$$

Once the EW symmetry is broken by the Higgs vevs, $v_{H_{1}}$ and $v_{H_{2}}$ with $v_{\mathrm{EW}}^{2}=v_{H_{1}}^{2}+v_{\mathrm{H}_{2}}^{2}=246 \mathrm{GeV}$, the Dirac mass matrices are simply given by

$$
M_{i j}^{e}=\frac{v_{H_{2}}}{\sqrt{2}} c_{i j}^{e} \epsilon^{n_{i j}^{e}}, \quad M_{i j}^{\nu}=\frac{v_{H_{2}}}{\sqrt{2}} c_{i j}^{\nu} \epsilon^{n_{i j}^{\nu}} .
$$

At leading order, the charged lepton masses are

$$
\frac{m_{e}}{m_{\tau}}=\frac{\left(c_{12}^{e}-c_{23}^{e}\right)^{2}}{c_{23}^{e^{2}}-1} \epsilon^{4}, \quad \frac{m_{\mu}}{m_{\tau}}=\left(1-c_{23}^{e^{2}}\right) \epsilon^{2} .
$$

Taking $\epsilon=0.1$, the following matrix of $c_{i j}^{e}$ coefficients reproduce the correct hierarchy between generations:

$$
c_{i j}^{e}=\left(\begin{array}{ccc}
1.0 & 1.6 & 1.0 \\
1.6 & 1.0 & -2.7 \\
1.0 & -2.7 & 1.0
\end{array}\right)
$$

Since the tau mass is not suppressed by any additional factor, we expect $v_{\mathrm{H}_{2}} \simeq \epsilon^{2} v_{\mathrm{EW}}$. For this hierarchical scenario, the mixing pattern is 


$$
\left|U_{L}^{e}\right|_{i j}=\left|U_{R}^{e}\right|_{i j} \approx \delta_{i j}+\epsilon^{n_{i j}^{e}} / \epsilon^{n_{j j}^{e}} \quad \text { with } i \leq j .
$$

Then, the $e-\mu$ mixing is $\mathcal{O}(\epsilon) \sim 0.1$.

The masses of the active neutrinos are produced through the usual type-I seesaw mechanism. Notice that, in this kind of formulations, the Pontecorvo-Maki-Nakagawa-Sakata (PMNS) matrix can always be achieved by a proper structure of the $M_{R}$ matrix [57].

After the breaking of the flavor symmetry, the flavon field can be parametrized as

$$
\phi(x)=\frac{1}{\sqrt{2}}\left(v_{\phi}+s(x)\right) e^{i a(x) / v_{\phi}},
$$

with $s(x)$ a $C P$-even scalar and $a(x)$ the ALP. If all the interactions respect the $\mathrm{U}(1)_{\phi} \times Z_{2}$ symmetry, $a(x)$ is the massless Nambu-Goldstone boson emerging after the spontaneous breaking. In our model, however, a contribution to the mass of the ALP comes from the explicit soft breaking of the symmetry in Eq. (3),

$$
V \supset V_{\text {soft }}=m^{2} H_{1} \cdot H_{2}+\text { H.c., }
$$

With the help of the $\mathrm{U}(1)_{\phi}$ symmetry, the ALP field can be removed from all the terms in the Lagrangian through a rotation $e^{i Q_{f} a / v_{\phi}}$, except for the kinetic terms and this soft-breaking term. Thus, after EWSB, the soft-breaking interaction becomes

$$
V_{\text {soft }}=m^{2} v_{H_{1}} v_{H_{2}} \cos \left(2 a(x) / v_{\phi}\right) .
$$

Expanding the cosine, we obtain naturally,

$$
m_{a}^{2} \simeq 4 m^{2} \frac{v_{H_{1}} v_{H_{2}}}{f_{a}^{2}} \sim \mathcal{O}(1) \mathrm{keV}^{2},
$$

where $f_{a} \sim \mathcal{O}\left(v_{\phi}\right) \sim 10^{9} \mathrm{GeV}, m \sim \mathcal{O}(\mathrm{EW}), v_{H_{1}} \sim v_{\mathrm{EW}}$ and, as stated before, $v_{\mathrm{H}_{2}} \sim \varepsilon^{2} v_{\mathrm{EW}}$. Alternatively, one may assume a hidden strong sector coupled to the ALP that generates its mass. In the following, we treat the ALP mass as a free parameter and, as preferred by the Xenon1T data, it should be around the $\mathrm{keV}$ scale.

The interaction between the pseudo-Nambu-Goldstone boson and the charged leptons, in the mass basis, is

$$
-\mathcal{L}_{a e}=i \frac{\partial_{\mu} a}{2 f_{a}} \bar{e}_{i} \gamma^{\mu}\left(V_{i j}^{e}+\gamma^{5} A_{i j}^{e}\right) e_{j}
$$

The axial and vector couplings in Eq. (14) are defined as ${ }^{1}$

$$
V_{i j}^{e}=\frac{1}{2}\left(U_{R}^{e \dagger} x_{R} U_{R}^{e}+U_{L}^{e \dagger} x_{L} U_{L}^{e}\right),
$$

\footnotetext{
${ }^{1}$ For $i=j$, we can always redefine the fields to have $V_{i i}^{e}=0[58]$.
}

$$
A_{i j}^{e}=\frac{1}{2}\left(U_{R}^{e \dagger} x_{R} U_{R}^{e}-U_{L}^{e \dagger} x_{L} U_{L}^{e}\right),
$$

with $x_{L}$ and $x_{R}$ the diagonal $3 \times 3$ matrices whose elements are the charged-lepton $\mathrm{U}(1)_{\phi}$ charges and $U_{L}^{e}, U_{R}^{e}$ the unitary transformations that diagonalize the mass matrices. ${ }^{2}$ In general, Eqs. (15) and (16) induce FV effects which are subject to constraints from different experiments, as it is discussed in Sec. III.

\section{B. Model II: general mixing}

In model II, we generalize the previous structure to allow for arbitrary leptonic mixings. To do this, we consider the $\mathrm{U}(1)_{\phi}$ global symmetry as only part of a larger flavor symmetry, $\mathcal{F}$, that will determine the Yukawa structure with the observed hierarchy among generations in the lepton sector. In this way, the $\mathrm{U}(1)_{\phi}$ symmetry remains flavor dependent, but masses and mixings are not fixed by the $\mathrm{U}(1)_{\phi}$ charges.

As an example, we use the same $\mathrm{U}(1)_{\phi} \times Z_{2}$ charges as in model I although now we can take $v_{\phi} / \Lambda \simeq \mathcal{O}(1)$. The scalar potential and Yukawa terms remain as in Eqs. (3) and (4) but, in this case, we highlight that the coefficients $c_{i j}^{e}$ and $c_{i j}^{\nu}$ are NOT forced to be $\mathcal{O}(1)$.

Adjusting them, different mixing patterns can be obtained. In particular, we are interested in the case of large PMNS-like mixing for charged leptons. As a typical benchmark model, we assume that the breaking of the symmetry $\mathcal{F}$ produces charged-lepton Yukawa couplings with PMNS-like mixing in the left- and right-handed sector. The couplings with the ALP are determined by Eqs. (15) and (16), but now

$$
V_{i j}^{e}, A_{i j}^{e}=\frac{1}{2} U_{\mathrm{PMNS}}^{e \dagger}\left(x_{R} \pm x_{L}\right) U_{\mathrm{PMNS}}^{e} .
$$

Then, for example, we can deduce the size of the axial 12coupling to be as large as $A_{12}^{e} \simeq 0.56$. Note that $V_{i j}^{e}=0$ by construction since $x_{L}=-x_{R}$.

\section{CONSTRAINTS FROM LFV AND ASTROPHYSICS}

Non-universal charges of the charged leptons under the $\mathrm{U}(1)_{\phi}$ global symmetry, together with nontrivial rotations to the mass basis, imply FV interactions between the ALP and these fermions. The absence of the anomalous coupling between the ALP and photons at tree level makes the search for ALPs by charged-lepton flavor-violating processes specially relevant. Detailed discussions about the phenomenology of flavorful ALPs can be found in $[58,59]$.

\footnotetext{
${ }^{2}$ In our convention: $U_{L}^{e^{\dagger}} M_{e} U_{R}^{e}=\operatorname{Diag}\left(m_{e}, m_{\mu}, m_{\tau}\right)$.
} 
TABLE I. Limits over the axion decay constant from lepton decays. Belle-II limits are derived from the simulated result at Belle [60] by rescaling the luminosity [58].

\begin{tabular}{lcc}
\hline \hline Lepton decay & BR limit & \multicolumn{1}{c}{ Experiment } \\
\hline \multicolumn{3}{c}{ Present best limits } \\
$\operatorname{BR}(\mu \rightarrow e a)$ & $<2.6 \times 10^{-6}$ & Jodidio et al. [61] \\
$\operatorname{BR}(\mu \rightarrow e a)$ & $<2.1 \times 10^{-5}$ & TWIST [62] \\
$\operatorname{BR}(\mu \rightarrow e a \gamma)$ & $<1.1 \times 10^{-9}$ & Crystal Box [63] \\
$\operatorname{BR}(\tau \rightarrow e a)$ & $<2.7 \times 10^{-3}$ & ARGUS [64] \\
$\operatorname{BR}(\tau \rightarrow \mu a)$ & $<4.5 \times 10^{-3}$ & ARGUS [64] \\
& Projections of running experiments \\
$\operatorname{BR}(\mu \rightarrow e a)$ & $<1.3 \times 10^{-7}$ & MEGII-fwd [58,65] \\
$\operatorname{BR}(\tau \rightarrow e a)$ & $<8.4 \times 10^{-6}$ & Belle-II \\
$\operatorname{BR}(\tau \rightarrow \mu a)$ & $<1.6 \times 10^{-5}$ & Belle-II \\
& Projections of planned experiments \\
$\operatorname{BR}(\mu \rightarrow e a)$ & $<7.3 \times 10^{-8}$ & Mu3e [66] \\
\hline \hline
\end{tabular}

Table I collects the experimental present limits and projected sensitivities for the search of ALPs through the detection of the FV process $\ell_{i} \rightarrow \ell_{j} a$. For an ALP mass around the $\mathrm{keV}$, the branching ratio for the FV transition $\ell_{i} \rightarrow \ell_{j} a$ is given by

$$
\operatorname{BR}\left(\ell_{i} \rightarrow \ell_{j} a\right)=\frac{m_{\ell_{i}}^{3}}{16 \pi \Gamma\left(\ell_{j}\right)} \frac{\left|C_{i j}^{e}\right|^{2}}{4 f_{a}^{2}}\left(1-\frac{m_{a}^{2}}{\ell_{i}^{2}}\right)^{2},
$$

with $\left|C_{i j}^{e}\right|^{2}=\left|V_{i j}^{e}\right|^{2}+\left|A_{i j}^{e}\right|^{2}$. For a given model, where the interaction between the ALP and charged leptons is fixed, the bounds in Table I on the $\ell_{i} \rightarrow \ell_{j} a$ transitions can be translated into bounds on $f_{a}$. Although all of them have been inspected, the strongest limits come from $\mu \rightarrow \mathrm{e} a$.

Regarding astrophysics bounds, interesting limits can be derived from stellar evolution. In particular, the cooling of white dwarfs [67] and red giants [68,69] impose strong constraints over the ALP interactions to matter and radiation. For massless ALP, the limits at 95\% C.L. are

$$
\begin{aligned}
& f_{a} \gtrsim 2.3 \times 10^{9}\left|C_{11}^{e}\right| \mathrm{GeV}, \\
& f_{a} \gtrsim 1.2 \times 10^{9}\left|C_{11}^{e}\right| \mathrm{GeV} .
\end{aligned}
$$

For ALP masses above $1 \mathrm{keV}$, the cooling rate is Boltzmann suppressed and the limits above should be rescaled by the factor $\sqrt{\xi\left(m_{a}, T\right) / \xi(0, T)}$, where [69]

$$
\xi\left(m_{a}, T\right)=\frac{1}{2 \pi^{2}} \int_{m_{a}}^{\infty} \frac{E^{2} \sqrt{E^{2}-m_{a}^{2}}}{e^{E / T}-1} .
$$

Finally, we comment on an additional bound from the big bang nucleosynthesis (BBN). As discussed in [70], by constraining the effective number of relativistic neutrino species during BBN, non-negligible limits can be set over the lepton-ALP coupling that compete with those from stellar evolution. When $m_{a} \sim[20,1000] \mathrm{keV}$, the BBN bound is indeed the strongest one. In our scenario, however, with a few keV ALP mass, stellar evolution remains the most important phenomena.

\section{RESULTS}

In [2], the authors conclude that an ALP satisfying

$$
A_{11}^{e} \simeq 10^{-13} \frac{f_{a}}{m_{e}}, \quad \text { for } m_{a} \in[2,3] \mathrm{keV}
$$

can reproduce the Xenon1T signal together with some reported anomalies in stellar cooling [67,69,71]. Such possibility can be realized in the context of anomaly-free DM ALPs, provided that they constitute only around the $10 \%$ of the total DM abundance $\left(\Omega_{a} / \Omega_{\mathrm{DM}} \sim 0.1\right)$.

Regarding the production of this anomaly-free ALP in the early Universe, two main mechanisms can be discussed: thermal and nonthermal production. The former is due to scatterings between leptons and the Higgs particles in the thermal plasma, and would require a reheating temperature $\sim \mathcal{O}\left(10^{2} \mathrm{GeV}\right)$, together with $m_{H_{2}} \sim \mathcal{O}\left(10^{2} \mathrm{GeV}\right)$. Such a relative low reheating temperature would not allow for thermal leptogesis, but other baryon generation mechanisms as Affleck-Dine [72] or resonant leptogenesis [73] would still be possible in a supersymmetric scenario. However, thermally produced warm DM is subject to important constraints from structure formation that might be in tension with the ALP masses considered here. In particular, the Lyman- $\alpha$ limit establishes that $m_{a} \geq 15 \mathrm{keV}$, if $\Omega_{a}=\Omega_{\mathrm{DM}}$ [74,75]. Although this bound is expected to be relaxed for our ALPs with $\Omega_{a} / \Omega_{\mathrm{DM}} \simeq 0.1$ [76], it is not straightforward to rescale this constraint with the DM fraction and we leave the detailed computation for a future work.

Nonthermal production, on the other hand, can safely evade these constraints. We can use the so-called misalignment mechanism, where the ALP starts oscillating when the Hubble parameter is comparable to its mass $[2,71]$ or we can also consider the ALP is produced by the decay of the inflaton. In both cases, a low reheating temperature is needed to avoid the thermal production of the ALP.

The discussion in $[2,71]$ is restricted to astrophysical and cosmological constraints while flavor observables are not discussed. Here, we aim to highlight the role of flavor observables to (dis)prove this kind of models.

Figures 1 and 2 show the Xenon1T favored prediction for $f_{a}$, based on the result in Eq. (22) (black diamond). Similarly, current and expected sensitivity from Jodidio et al. [61] (green continuous line) and MU3E [66] (red dashed line) in dedicated searches for $\mu \rightarrow$ e $\gamma$ are displayed as a function of $m_{a}$. We also show the projection of the proposal by Calibbi et al. [58], MEGII-fwd (yellow dashed line), for MEGII [65] to improve the detection of the 


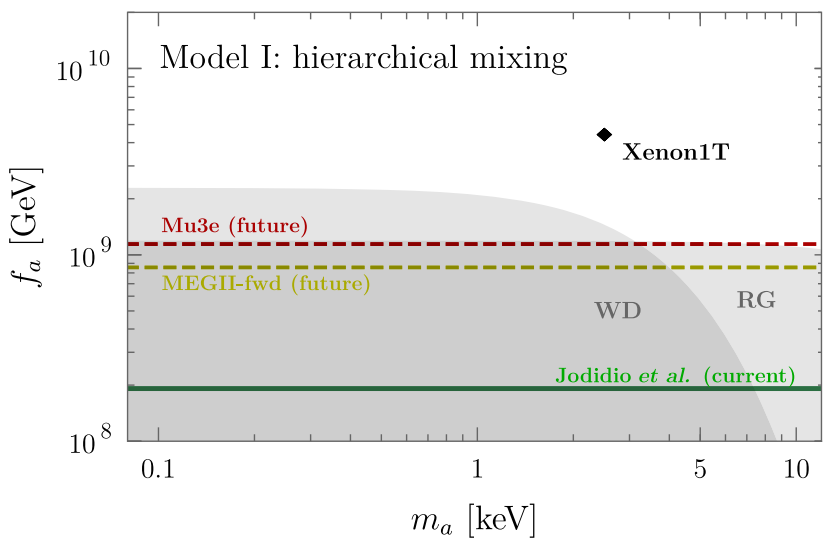

FIG. 1. Results for model I with hierarchical Yukawa couplings generated à la Froggatt-Nielsen.

process of interest, $\mu \rightarrow e a$. Finally, limits due to white dwarfs and red giants (gray shaded regions) also impose relevant bounds on our models [58].

From Fig. 1, we notice that testing model I (small mixing) with LFV observables remains quite challenging, even for future sensitivities. On the other hand, scenarios with larger mixing effects in the charged-lepton sector provide better prospects. For model II, in Fig. 2, we observe that while current limits are not sufficient to constrain the model, more stringent bounds coming from MU3E or the implementation of MEGII-fwd are enough to probe this formulation. One may then conclude that LFV can clearly complement astrophysics searches and, in some cases, go beyond them. Flavored ALP models provide a rich phenomenology to be investigated with present and future data.

Regarding the Higgs sector of our models, we briefly comment on its phenomenology here. A type-X 2HDM has been considered in our realizations, where $H_{1}$ couples to quarks and $\mathrm{H}_{2}$ to leptons. In the absence of $\mathrm{CP}$ violation at tree level, the Higgs spectrum consist of two scalars, one pseudoscalar and two charged states. Our formulations are defined in the large $\tan \beta=v_{H_{1}} / v_{H_{2}}$ regime, where

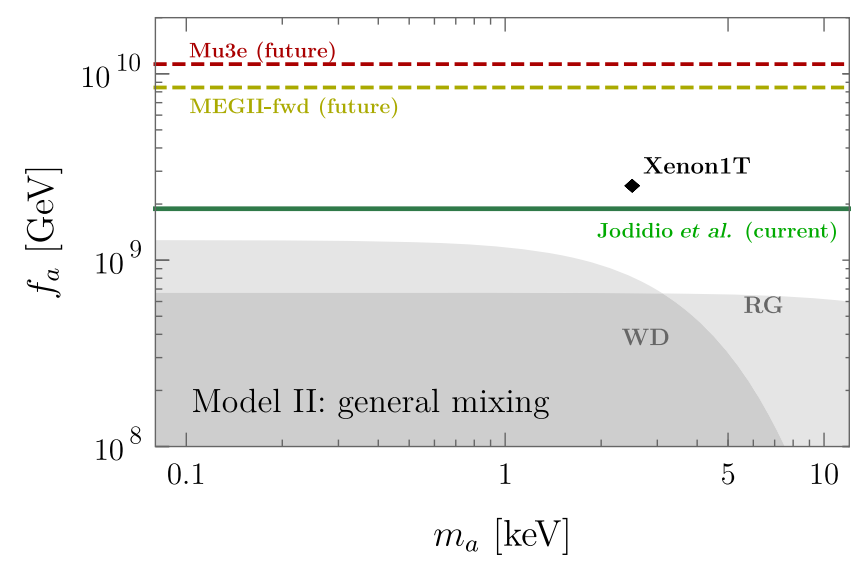

FIG. 2. Results for model II with general Yukawa matrices and mixing. the pseudoscalar and the charged Higgses are essentially given by the leptophilic Higgs. We also assume the decoupling limit, where $\sin (\beta-\alpha) \simeq 1$ and the lightest scalar behaves as the SM Higgs and the observed mass can be reproduced with a mild tuning of the parameters in the Higgs potential.

The main constraints to this scenario are those on the mass of the charged Higgses and come from LEP, which establishes the model independent limit $M_{H^{ \pm}}>79.3 \mathrm{GeV}$ [77], the tau decay $\tau \rightarrow \mu \bar{\nu} \nu$ [78], the charged Higgs decay $H^{ \pm} \rightarrow \tau^{ \pm} \nu$ and the top decay $t \rightarrow H^{+} b$, if $M_{H^{+}} \lesssim m_{t}-m_{b}$ [79]. The latter two bounds apply for $\tan \beta \lesssim 17$, so they are safely evaded in our case [80]. Similarly, our leptophilic Higgs states escape the strong constraints over the charged Higgs masses due to $B \rightarrow X_{s} \gamma$ [81] and to $B \rightarrow \tau \nu$ [82], common in type-II 2HDM [55]. The tau decay, in contrast, imposes the following limit for the considered $\tan \beta$ regime: $M_{H^{ \pm}} \gtrsim 200 \mathrm{GeV}$ [55].

Regarding neutral states, we have a mild bound over the pseudoscalar mass, $M_{A}>10 \mathrm{GeV}$, from $B_{s} \rightarrow \mu \mu$ [83]. LHC searches of heavy neutral Higgses in the dilepton [84] and ditau $[85,86]$ final state do not introduce relevant constraints in our model, mainly due to their limited $H$ and $A$ production in this environment with large $\tan \beta$ and small $\cos (\beta-\alpha)$, where both the gluon and weak-boson fusion production are strongly suppressed [55,87]. Therefore, present bounds on extra Higgses are easily satisfied in our model taking a scalar mass in the potential $m \gtrsim 200 \mathrm{GeV}$, of the order of the electroweak scale.

\section{CONCLUSIONS}

In this paper, we considered the LFV effects from a keV scale flavor-dependent ALP which is motivated by recent Xenon1T results. We find that, for a general mixing in the lepton sector, the leptonic flavor changing experiments could confirm or exclude the possibility of explaining the Xenon1T result by an ALP, while being consistent with all phenomenological and astrophysical constraints. On the other hand, if the leptonic mixing originating from the Froggatt-Nielsen symmetry are small, CKM-like, the measurement of their LFV effects would constitute a challenge for future experiments.

\section{ACKNOWLEDGMENTS}

The authors thank Arcadi Santamaria for useful discussions. C. H. acknowledges support from the Sun Yat-Sen University Science Foundation. M. L. L. I. acknowledges support from the China Postdoctoral Science Foundation Grant No. 2020M670475. A. M. acknowledges support from La-Caixa-Severo Ochoa scholarship. A. M. and O. V. are supported by Spanish and European funds under Ministerio de Ciencia, Innovacion y Universidades (MICIU) Grant No. FPA2017-84543-P. O. V. acknowledges partial support from the "Generalitat Valenciana" Grant No. PROMETEO2017-033. J. M. Y. acknowledges funding 
from the National Natural Science Foundation of China (NNSFC) under Grants No. 12075300, No. 11821505, and No. 11851303, from Peng-Huan-Wu Theoretical Physics Innovation Center (12047503), from the CAS Center for
Excellence in Particle Physics (CCEPP), from the CAS Key

Research Program of Frontier Sciences and from a Key R\&D Program of Ministry of Science and Technology under Grant No. 2017YFA0402204.
[1] E. Aprile et al. (XENON Collaboration), arXiv:2006.09721.

[2] F. Takahashi, M. Yamada, and W. Yin, arXiv:2006.10035.

[3] I. M. Bloch, A. Caputo, R. Essig, D. Redigolo, M. Sholapurkar, and T. Volansky, arXiv:2006.14521.

[4] J. B. Dent, B. Dutta, J. L. Newstead, and A. Thompson, arXiv:2006.15118.

[5] G. Cacciapaglia, C. Cai, M. T. Frandsen, M. Rosenlyst, and H. H. Zhang, arXiv:2006.16267.

[6] T. Li, arXiv:2007.00874.

[7] H. N. Long, D. V. Soa, V. H. Binh, and A. E. Cárcamo Hernández, arXiv:2007.05004.

[8] P. Athron, C. Balázs, A. Beniwal, J. E. Camargo-Molina, A. Fowlie, T. E. Gonzalo, S. Hoof, F. Kahlhoefer, D. J. E. Marsh, M. T. Prim, P. Scott, W. Su, M. White, L. Wu, and Y. Zhang, arXiv:2007.05517.

[9] D. Croon, S.D. McDermott, and J. Sakstein, arXiv: 2007.00650.

[10] D. Croon, S. D. McDermott, and J. Sakstein, arXiv: 2007.07889.

[11] K. Kannike, M. Raidal, H. Veermäe, A. Strumia, and D. Teresi, arXiv:2006.10735.

[12] G. Alonso-Álvarez, F. Ertas, J. Jaeckel, F. Kahlhoefer, and L. J. Thormaehlen, arXiv:2006.11243.

[13] B. Fornal, P. Sandick, J. Shu, M. Su, and Y. Zhao, arXiv: 2006.11264.

[14] K. Harigaya, Y. Nakai, and M. Suzuki, arXiv:2006.11938.

[15] L. Su, W. Wang, L. Wu, J. M. Yang, and B. Zhu, arXiv: 2006.11837.

[16] M. Du, J. Liang, Z. Liu, V. Tran, and Y. Xue, arXiv: 2006.11949.

[17] N. F. Bell, J. B. Dent, B. Dutta, S. Ghosh, J. Kumar, and J. L. Newstead, arXiv:2006.12461.

[18] Y. Chen, J. Shu, X. Xue, G. Yuan, and Q. Yuan, arXiv: 2006.12447.

[19] U. K. Dey, T. N. Maity, and T. S. Ray, arXiv:2006.12529.

[20] G. Choi, M. Suzuki, and T. T. Yanagida, arXiv:2006.12348.

[21] G. Paz, A. A. Petrov, M. Tammaro, and J. Zupan, arXiv: 2006.12462.

[22] Q. H. Cao, R. Ding, and Q. F. Xiang, arXiv:2006.12767.

[23] R. Primulando, J. Julio, and P. Uttayarat, arXiv:2006.13161.

[24] K. Nakayama and Y. Tang, arXiv:2006.13159.

[25] Y. Jho, J. C. Park, S. C. Park, and P. Y. Tseng, arXiv:2006 .13910 .

[26] J. Bramante and N. Song, arXiv:2006.14089.

[27] M. Baryakhtar, A. Berlin, H. Liu, and N. Weiner, arXiv: 2006.13918.

[28] H. An, M. Pospelov, J. Pradler, and A. Ritz, arXiv:2006 .13929 .
[29] L. Zu, G. W. Yuan, L. Feng, and Y.Z. Fan, arXiv:2006 .14577 .

[30] K. Zioutas, G. Cantatore, M. Karuza, A. Kryemadhi, M. Maroudas, and Y. K. Semertzidis, arXiv:2006.16907.

[31] H. An and D. Yang, arXiv:2006.15672.

[32] L. Delle Rose, G. Hütsi, C. Marzo, and L. Marzola, arXiv: 2006.16078.

[33] G. Arcadi, A. Bally, F. Goertz, K. Tame-Narvaez, V. Tenorth, and S. Vogl, arXiv:2007.08500.

[34] D. Choudhury, S. Maharana, D. Sachdeva, and V. Sahdev, arXiv:2007.08205.

[35] G. Choi, T. T. Yanagida, and N. Yokozaki, arXiv:2007 .04278 .

[36] C. W. Chiang and B. Q. Lu, arXiv:2007.06401.

[37] H. J. He, Y. C. Wang, and J. Zheng, arXiv:2007.04963.

[38] I. M. Shoemaker, Y. D. Tsai, and J. Wyenberg, arXiv:2007 .05513.

[39] C. Boehm, D. G. Cerdeno, M. Fairbairn, P. A. N. Machado, and A.C. Vincent, arXiv:2006.11250.

[40] A. Bally, S. Jana, and A. Trautner, arXiv:2006.11919.

[41] D. Aristizabal Sierra, V. De Romeri, L. J. Flores, and D. K. Papoulias, arXiv:2006.12457.

[42] J. Buch, M. A. Buen-Abad, J. Fan, and J. S. C. Leung, arXiv:2006.12488.

[43] A. N. Khan, arXiv:2006.12887.

[44] S. F. Ge, P. Pasquini, and J. Sheng, arXiv:2006.16069.

[45] K. S. Babu, S. Jana, and M. Lindner, arXiv:2007.04291.

[46] C. Gao, J. Liu, L. T. Wang, X. P. Wang, W. Xue, and Y. M. Zhong, arXiv:2006.14598.

[47] R. Budnik, H. Kim, O. Matsedonskyi, G. Perez, and Y. Soreq, arXiv:2006.14568.

[48] P. Coloma, P. Huber, and J. M. Link, arXiv:2006.15767.

[49] L. Di Luzio, M. Fedele, M. Giannotti, F. Mescia, and E. Nardi, arXiv:2006.12487.

[50] W. DeRocco, P. W. Graham, and S. Rajendran, arXiv: 2006.15112.

[51] C. Dessert, J. W. Foster, Y. Kahn, and B. R. Safdi, arXiv: 2006.16220.

[52] C. D. Froggatt and H. B. Nielsen, Nucl. Phys. B147, 277 (1979).

[53] Y. Ema, K. Hamaguchi, T. Moroi, and K. Nakayama, J. High Energy Phys. 01 (2017) 096.

[54] L. Calibbi, F. Goertz, D. Redigolo, R. Ziegler, and J. Zupan, Phys. Rev. D 95, 095009 (2017).

[55] M. Aoki, S. Kanemura, K. Tsumura, and K. Yagyu, Phys. Rev. D 80, 015017 (2009).

[56] E. J. Chun, S. Dwivedi, T. Mondal, B. Mukhopadhyaya, and S. K. Rai, Phys. Rev. D 98, 075008 (2018). 
[57] A. Masiero, S. K. Vempati, and O. Vives, Nucl. Phys. B649, 189 (2003).

[58] L. Calibbi, D. Redigolo, R. Ziegler, and J. Zupan, arXiv:2006.04795.

[59] F. Björkeroth, E. J. Chun, and S. F. King, J. High Energy Phys. 08 (2018) 117.

[60] K. Griessinger (BABAR Collaboration), Nucl. Part. B, Phys. Proc. 287-288, 47 (2017).

[61] A. Jodidio, B. Balke, J. Carr, G. Gidal, K. A. Shinsky, H. M. Steiner, D. P. Stoker, M. Strovink, R. D. Tripp, B. Gobbi, and C.J. Oram, Phys. Rev. D 34, 1967 (1986).

[62] R. Bayes et al. (TWIST Collaboration), Phys. Rev. D 91, 052020 (2015).

[63] R. D. Bolton et al., Phys. Rev. D 38, 2077 (1988).

[64] H. Albrecht et al. (ARGUS Collaboration), Z. Phys. C 68, 25 (1995).

[65] A. M. Baldini et al. (MEG II Collaboration), Eur. Phys. J. C 78, 380 (2018).

[66] A. K. Perrevoort, https://doi.org/10.11588/heidok.00024585.

[67] M. M. Miller Bertolami, B. E. Melendez, L. G. Althaus, and J. Isern, J. Cosmol. Astropart. Phys. 10 (2014) 069.

[68] G. Raffelt and A. Weiss, Phys. Rev. D 51, 1495 (1995).

[69] N. Viaux, M. Catelan, P. B. Stetson, G. Raffelt, J. Redondo, A. A. R. Valcarce, and A. Weiss, Phys. Rev. Lett. 111, 231301 (2013).

[70] D. Ghosh and D. Sachdeva, arXiv:2007.01873.

[71] K. Nakayama, F. Takahashi, and T. T. Yanagida, Phys. Lett. B 734, 178 (2014).

[72] I. Affleck and M. Dine, Nucl. Phys. B249, 361 (1985).
[73] A. Pilaftsis and T. E. J. Underwood, Nucl. Phys. B692, 303 (2004).

[74] V. Iršič, M. Viel, M. G. Haehnelt, J. S. Bolton, S. Cristiani, G. Cupani, T. S. Kim, V. D’Odorico, S. López, S. Ellison et al. Phys. Rev. D 96, 023522 (2017).

[75] A. Kamada and K. Yanagi, J. Cosmol. Astropart. Phys. 11 (2019) 029.

[76] R. Diamanti, S. Ando, S. Gariazzo, O. Mena, and C. Weniger, J. Cosmol. Astropart. Phys. 06 (2017) 008.

[77] A. Heister et al. (ALEPH Collaboration), Phys. Lett. B 543, 1 (2002).

[78] P. A. Zyla et al. (Particle Data Group), Prog. Theor. Exp. Phys. 2020, 083C01 (2020).

[79] M. Aaboud et al. (ATLAS Collaboration), J. High Energy Phys. 09 (2018) 139.

[80] P. Sanyal, Eur. Phys. J. C 79, 913 (2019).

[81] A. Abdesselam et al. (Belle Collaboration), arXiv:1608 .02344 .

[82] Y.S. Amhis et al. (HFLAV Collaboration), arXiv:1909. 12524.

[83] L. Wang and X. F. Han, J. High Energy Phys. 05 (2015) 039.

[84] M. Aaboud et al. (ATLAS Collaboration), J. High Energy Phys. 10 (2017) 182.

[85] M. Aaboud et al. (ATLAS Collaboration), J. High Energy Phys. 01 (2018) 055.

[86] V. Khachatryan et al. (CMS Collaboration), J. High Energy Phys. 02 (2017) 048.

[87] G. C. Branco, P. M. Ferreira, L. Lavoura, M. N. Rebelo, M. Sher, and J. P. Silva, Phys. Rep. 516, 1 (2012). 